\title{
Effect of coarse particle volume fraction on the yield stress and thixotropy of cementitious materials
}

\author{
Fabien Mahaut ${ }^{\mathrm{a}}$, Samir Mokéddem ${ }^{\mathrm{a}}$, Xavier Chateau ${ }^{\mathrm{a}}$, \\ Nicolas Roussel ${ }^{b}$, Guillaume Ovarlez ${ }^{\mathrm{a}, *}$ \\ ${ }^{a}$ Université Paris Est - Institut Navier - Laboratoire des Matériaux et Structures \\ du Génie Civil (LCPC-ENPC-CNRS) - Champs-sur-Marne, France ${ }^{1}$ \\ ${ }^{\mathrm{b}}$ Université Paris Est - Laboratoire Central des Ponts et Chaussées, Paris, France.
}

\begin{abstract}
In order to help modelling the yield stress of fresh concrete, we study the behavior of suspensions of coarse particles in a thixotropic cement paste. Our aim is to relate the yield stress of these mixtures to the yield stress of the suspending cement paste, to the time passed at rest, and to the coarse particle volume fraction. We present here procedures that allow for (i) studying an homogeneous and isotropic suspension, (ii) comparing the yield stress of a given cement paste to that of the same cement paste added with particles, (iii) accounting for the thixotropy of the cement paste. We observe that the yield stress of these suspensions of cement paste with coarse particles follows the very simple Chateau-Ovarlez-Trung model [1], consistently with the experimental results of Mahaut et al. [2] obtained with many different particles and suspending yield stress fluids. This consistency between the results obtained in various yield stress fluids shows that the yield stress of the suspension does not depend on the physicochemical properties of the suspending yield stress fluid; it only
\end{abstract}


depends on its yield stress value. This shows that studies of suspensions in model yield stress fluids can be used as a general tool to infer the behavior of fresh concrete. Moreover, we show that the thixotropic structuration rate of the interstitial paste (its static yield stress increase rate in time) is not affected by the presence of the particles. As a consequence, it is sufficient to measure the thixotropic properties of the constitutive cement paste in order to predict the thixotropic structuration rate of a given fresh concrete. This structuration rate is predicted to have the same dependence on the coarse particle volume fraction as the yield stress.

Key words: A. Fresh Concrete, A. Rheology, D. Aggregate, D. Cement Paste, E. Modeling

\section{$1 \quad 1 \quad$ Introduction}

2 Knowing and predicting the flow properties of fresh concrete is a major issue of 3 concrete casting and concrete mix-design. Basically, fresh concretes exhibit a 4 yield stress [3] and have a solid viscoelastic behavior below this yield stress [4]; 5 above the yield stress they behave as liquids, and their steady flow behavior 6 is usually well represented by a Bingham or a Herschel-Bulkley model $[3,5]$. 7 However, fresh concrete is also known for its evolving rheological behavior. 8 Even, if its steady state flow may be described by the above models, the 9 characteristic time to reach this steady state flow may be rather long [6-9] and, after a long time of rest, the stress that has to be applied to induce a flow may be one or two orders higher than the dynamic yield stress measured when the

* corresponding author: guillaume.ovarlez@lcpc.fr

1 Support from the Agence Nationale de la Recherche (ANR) is acknowledged (grant ANR-05-JCJC-0214). 
material stops flowing i.e. it is thixotropic [10-12]. The static yield stress and its increase rate at rest are actually the most important rheological quantities in terms of potential applications in the case of SCC [13]: it has been shown recently that they determine the formwork pressure $[9,14-17]$, the stability vs. sedimentation of the coarsest particles in SCC [18] and the occurrence of distinct layer casting [19]. As a consequence it is of high importance to understand the role of the various components of a given concrete on this yield stress and its evolution at rest. Moreover, measuring directly the rheological properties of fresh concrete is very difficult [20]; any model providing the yield stress of concrete as a function of the suspending cement paste properties and the properties and the volume fraction of sand and aggregates would then prove to be very useful.

The link between concrete mix-design and its flow properties in the fresh state may be studied in the more general framework of suspensions rheophysics [12]. Actually, fresh concretes belong to the wide family of dense suspensions, which often involve a broad range of particle sizes [21] and can be found in many industrial processes (drilling muds, foodstuff transport...) and natural phenomena (debris-flows, lava flows...). All these materials share the same complex features, which originate from the great variety of interactions between the particles (colloidal, hydrodynamic, frictional, collisional...) and of physical properties of the particles (volume fraction, sensitivity to thermal agitation, shape...) affecting the material behavior $[22,12]$. Basically, in the absence of a contact network of noncollodial particles (i.e. for moderate noncollodial particles volume fraction), the yielding behavior originates from the colloidal interactions which create a jammed network of interacting particles $[5,12]$. Structuration at rest (which has nothing to do with setting) is observed 
in many aggregating suspensions and colloidal glasses [12]: the evolution of the behavior of aggregating suspensions at rest may be explained by a progressive and reversible formation of a solid structure by flocculation. Within this frame, the problem of the influence of coarse particles on the behavior of fresh concrete may be seen more generally as the problem of the influence of noncolloidal particles on the properties of yield stress fluids. It is thus of high importance to clarify the cases where the rheological properties of a suspension of coarse particles in a yield stress fluid depend only on the rheological properties of the suspending fluid and on the coarse particle volume fraction and size distribution. This should provide results applicable to any particles in any yield stress fluid, in particular to sand and aggregates suspended in a cement paste. It would allow the use of results obtained in studies performed e.g. with noncolloidal particles in clay dispersions to predict the behavior of a mortar or a concrete. On the other hand, any departure from generic results would be the result of specific physicochemical interactions in the suspensions (or specific slippage at the paste/particle interface), as e.g. the adsorption of a fraction of the superplasticizer of the cement paste on the fine aggregates in SCC [23], and would justify for each material a specific study with the particular particles and particular paste involved. In this paper, we test the idea of fresh concrete being a suspension of particles in a yield stress fluid. We compare the results obtained when suspending particles in a cement paste to those recently obtained in a broad range of materials (suspensions of various particles in various yield stress fluids) by Mahaut et al. [2].

The influence of the aggregates on the rheological properties of fresh concrete has been studied theoretically and experimentally by de Larrard [24], de Larrard and Sedran [25], Geiker et al. [26], Erdogan [27] and Toutou and Roussel 
[28]. De Larrard [24] has proposed a model in which concrete is looked as a granular mix in a water suspension. Then, the overall yield stress is the macroscopic counterpart of the friction between solid particles and is interpreted as the stress one needs to apply in order to overcome the intergranular contact forces. The overall yield stress can be estimated from the value of the solid volume fraction and close packing density of the different components of the granular mixture. However, if this model may help understanding the properties of fresh concrete displaying "ordinary" rheology, it is unadapted to the description of modern fluid concrete which contains less coarse particles and where friction between the grains is negligible [29]. Geiker et al. [26] have studied experimentally the effect of coarse particle volume fraction on the rheological properties of SCC. They have measured the steady-state flow curves of various materials thanks to the procedure developed in [8]; the dynamic yield stress was then extracted from a fit of the flow curve with a Bingham model. It was found to increase strongly with the coarse particle volume fraction. To model the behavior, they assume that the effect of aggregates on concrete rheological properties can be studied by looking to concrete as a suspension of coarse particles in the mortar seen as a continuum medium. Their experimental data are compared to a model proposed by Nielsen [30] which provides the yield stress of a suspension of ellipsoids as a function of the volume fraction of particles and of the aspect ratio. This model rests on heuristic rules which are not rigorously justified. Nevertheless, the theory can be calibrated in order to accurately describe the data of Geiker et al. [26]. Erdogan [27] have studied the effect of aggregate particle shape and surface texture on rheological properties of fresh concrete. Artificial aggregate particles of regular geometric shapes (spheres, cubes and rectangular prisms) with similar centimeter size and volume were prepared. A Couette-vane rheometer (ICAR) was used to 
measure the dynamic yield stress as the low shear rate limit of a flow curve. In addition, slump tests were performed. Erdogan has observed that the yield stress increases slightly when the coarse particle volume fraction increases. This trend is confirmed by slump experiments: the slump value was clearly a decreasing function of the coarse particle content, whatever the shape of the particles is. Toutou and Roussel [28] have studied the flow behavior of mortars, considered as suspensions of sand in a cement paste, and the flow behavior of concretes, considered as suspensions of gravel in a mortar. In both cases, the influence of the coarsest inclusions volume fraction on the suspending paste properties was investigated. The dynamic yield stress was extrapolated from the measured flow-curves. The yield stress of the mortar was found to increase with the sand volume fraction. However, at low volume fraction (below 20\%) yield stresses of mortars were found to be lower than the yield stress of the suspending cement paste. Toutou and Roussel [28] attributed this feature to the increased deflocculation of the cement paste due to the presence of the inclusions during mixing of the suspension, in agreement with Williams et al. [31]. The yield stress of concrete was also found to increase with the gravel volume fraction. However, Toutou and Roussel [28] found that adding gravel at a given volume fraction to a mortar yields a much larger increase of the yield stress than adding sand at the same volume fraction to a cement paste.

The influence of coarse particles on the rheological properties of other yield stress fluids has been studied by Coussot [32] and Ancey and Jorrot [33]. Ancey and Jorrot [33] have suspended coarse particles within a clay dispersion. They measured the yield stress of the suspension by means of a slump test. They showed that for well-graded particles, the suspension yield stress does not depend on the particle characteristics (diameter, material) and that the yield 
stress diverges when the solid volume fraction value tends toward the maximum packing fraction. Of course, when the coarse particles are polydisperse, the value of the maximum packing fraction depends on the size distribution of the particles, and the yield stress diverges for values of the solid volume fraction depending on this distribution. They observed sometimes that, for low reduced solid volume fraction, the yield stress can be a decreasing function of the solid volume fraction of the coarse particle. This effect was ascribed to a depletion phenomena: the clay particles are supposed to be expelled from the suspending fluid in the neighborhood of the coarse particles which are then embedded in a shell of pure water. Then, they cannot contribute to the overall yield stress: they behave as voids. Note that this depletion mechanism is specific to the suspending yield stress fluid studied by Ancey and Jorrot; thus, it cannot be used to predict what happens when the particles are suspended in another yield stress fluid.

The few existing experimental studies provide very different results; e.g., when particles having the same shape (spheres) are embedded at a volume fraction $\phi$ corresponding to $70 \%$ of the maximum packing fraction $\phi_{m}$ in a paste, Geiker et al. [26] find that the yield stress of the paste is increased by a factor 50 when the paste is a mortar, whereas Erdogan [27] finds that it is increased by only a factor 1.3 when the paste is also a mortar, and Ancey and Jorrot [33] find that, when the paste is a clay dispersion, the yield stress is increased by a factor 2. Other surprising discrepancies are shown by Toutou and Roussel [28]: they find that for sand suspended at $70 \%$ of $\phi_{m}$ in a cement paste the yield stress is increased by a factor 8 whereas for gravel (of comparable shape and dispersity) suspended at $70 \%$ of $\phi_{m}$ in a mortar, it is increased by a factor 25 . As pointed out above, if rigid noncolloidal particles of a given shape and dis- 
persity were to interact only rheologically with the suspending paste, we would expect all the results to be roughly consistent as they should not depend on the paste physicochemical nature. However, the discrepancy between the results of Geiker et al. [26], Erdogan [27], and Ancey and Jorrot[33], and between the mortar and the concrete case in the work of Toutou and Roussel [28], does not necessarily imply that there are specific physicochemical interactions between the different particles and the different pastes involved in these studies, and that we would fail describing these materials as suspensions of rigid particles in yield stress fluids. Such discrepancy may indeed find its origin in differences and shortcomings in the experimental procedures used. Actually, the experiments of Geiker et al. [26], Erdogan [27] and Toutou and Roussel [28] involve a flow of the material. It is then well known that shear-induced migration of particles towards low shear zones (the external cylinder in coaxial cylinders geometries) is likely to occur [34-36], whatever the care that is taken; this would cause the material to be heterogeneous inside the measurement cell, and the measurement to be non-representative of the homogeneous material. This is particularly true at high concentrations (above $50 \%$ for spherical monodisperse particles) where it has been shown by Ovarlez et al. [36] that radial migration occurs as an almost instantaneous and unavoidable process in a Couette geometry. In this case, all the measurements performed in time are likely to be performed on the same stationary heterogeneous structure: testing the material at the same rotational velocity at two different times [8] may then wrongly lead to conclude that there is no shear-induced migration while the only correct conclusion is that the structure is stationary. Moreover, Geiker et al. [26], Erdogan [27] and Toutou and Roussel [28] use a Herschel-Bulkley (or Bingham) fit of the flow curve to extrapolate the value of the yield stress instead of a direct measurement. Chateau et al. [1] have shown that such 
an extrapolation generally provides an overestimation of the yield stress of the suspension, and that this overestimation is more dramatic as the particle concentration increases. The reason is that the suspension departs from the Herschel-Bulkley (or Bingham) model at very low shear rate (unaccessible to most concrete rheometers) and has a lower yield stress than the one extrapolated from the measurable flow curve [1]. On the other hand, as the strain involved in this test is small, there should be no migration, nor extrapolation problems, in the slump test used by Ancey and Jorrot [33], as long as the yield stress is high enough to avoid spreading of the material and the correlation between measured slump and yield stress is suitable to their experiments [37]. Another difference between the procedures is that the particle distribution after a flow is anisotropic [38-40], whereas the particle distribution is hardly changed by the slump flow and is thus isotropic in the experiments of Ancey and Jorrot [33]; as a consequence, the results of Ancey and Jorrot [33] are not related to the same state of the suspension as the one of Erdogan [27] and Geiker et al. [26]. Finally, note that Ancey and Jorrot [33] and Toutou and Roussel [28] found in some cases that the suspension yield stress can be lower than the suspending paste yield stress; as pointed out by Chateau et al. [1], this should not occur if the noncolloidal particle interact only mechanically with the paste, i.e. these results are likely to apply only to their systems.

Finally, it is therefore of high importance to clarify the cases where suspensions can actually be considered as particles in a yield stress fluid, i.e. the cases where the rheological properties of the suspension depend only on the rheological properties of the suspending fluid and on the coarse particle volume fraction, shape and size distribution. With the aim of providing such generic results, Mahaut et al. [2] have recently performed an experimental study on 
a broad range of materials. They have suspended beads of various sizes and made of various materials in very different pastes whose common point is to exhibit a yield stress, and they sought consistency between the results. Moreover, they had a careful look at all the steps of the measurement procedure to ensure that an homogeneous and isotropic material is studied in all cases. They showed that the dimensionless elastic modulus $G^{\prime}(\phi) / G^{\prime}(0)$ and the dimensionless yield stress $\tau_{c}(\phi) / \tau_{c}(0)$ of such monodisperse suspensions depend on the bead volume fraction $\phi$ only (as expected for systems free from specific physicochemical interactions or specific slippage at the paste/particle interface). They found that the elastic modulus/concentration relationship is well fitted to a Krieger-Dougherty model $\left(1-\phi / \phi_{m}\right)^{-2.5 \phi_{m}}$ with $\phi_{m}=0.57$ for monodisperse isotropic suspensions. They showed that the yield stress/concentration relationship is related to the elastic modulus/concentration relationship through a very simple law $\tau_{c}(\phi) / \tau_{c}(0)=\sqrt{(1-\phi) G^{\prime}(\phi) / G^{\prime}(0)}$ in agreement with the micromechanical analysis of Chateau et al. [1], yielding the Chateau-OvarlezTrung model $\tau_{c}(\phi) / \tau_{c}(0)=\sqrt{(1-\phi)\left(1-\phi / \phi_{m}\right)^{-2.5 \phi_{m}}}$ for the yield stress of suspensions of monodisperse beads in a yield stress fluid.

In this paper, we study suspensions of coarse spherical particles in a thixotropic cement paste. We measure the static yield stress of the suspensions as a function of the resting time and of the particle volume fraction. We design new procedures that allow for comparing the yield stress of a given cement paste to that of the same cement paste added with particles. We also take care of designing a procedure that allows for properly accounting for thixotropy of the paste, independently of any irreversible change in the paste behaviour. In Sec. 2, we present the materials and the experimental setup. In Sec. 3, we present the procedure we developed to ensure comparing properly the yield 


\section{Materials and methods}

\subsection{Pastes and particles}

We performed our experiments with a thixotropic cement paste. White Cement CEM I/52.5 N CE CP2 NF "SB" from Gargenville Calcia was used to prepare all the cement pastes. Its specific gravity is 3.01. Its compressive strength is $62 \mathrm{MPa}$ at 28 days according to NF EN 196-1 test. The size distribution was measured in water using a laser granulometer (according to NF ISO 13320-1 test) for different amount of superplasticizer and is given in Fig. 1. The specific area determined using a BLAINE permeameter, according to NF EN 196-6 test, is $4117 \mathrm{~cm} 2 / \mathrm{g}$. The cement chemical constituents are summarized in Tab. 1. The Water to Cement ratio W/C studied here was 0.35. A Superplasticizer (Glenium 27) and a nanosilica slurry (Rhoximat CS 60 SL, Rhodia) were added to the mixture with a Superplasticizer to cement mass ratio of $1 \%$ and a nanosilica slurry to cement mass ratio of $2 \%$. The fluids (water + superplasticizer + nanosilica) were first mixed together to obtain an homogeneous suspension, and then added to the cement powder before a 5 minutes mixing phase in a planetary Controlab mixer: the velocity was first set to $140 \mathrm{rpm}$ during $2 \mathrm{~min}$, and then to $285 \mathrm{rpm}$ during $3 \mathrm{~min}$. All the exper- 
iments were performed on the fresh cement paste, less than $75 \mathrm{~min}$ since the constituents were mixed together. Before any measurement, the cement paste was presheared again in the mixer at $285 \mathrm{rpm}$ during $2 \mathrm{~min}$ in order to always start the experiments on a paste in an initially destructured state.

The particles suspended in the cement paste are spherical monodisperse glass beads of $2 \mathrm{~mm}$ diameter. This ensures that the particle size is much larger than the paste microstructure, so that the particles may "see" the cement paste as a continuum medium.

We chose to compare the results obtained with the suspensions of particles in a cement paste to the one obtained by Mahaut et al [2] where particles are suspended in various other yield stress fluids: emulsions, colloidal suspensions, and a physical gel (see Mahaut et al. [2] for details on the preparation of these materials). The emulsions are water in oil emulsions, in which the origin of the yield stress is the surface tension between the droplets [5]. The colloidal suspensions are bentonite suspensions, made of clay particles of length of order $1 \mu \mathrm{m}$ and thickness $10 \mathrm{~nm}$. The yield stress then originates from colloidal interactions between the particles. The physical gel is a Carbopol dispersion. Basically, the polymers arrange in roughly spherical blobs which are squeezed together $[41,42]$; this yields a yield stress. The particles used in the Mahaut et al. study are spherical monodisperse beads. They are either polystyrene beads of density 1.05, or glass beads of density 2.5., of various particle diameters: 80 , $140,315 \mu \mathrm{m}$ in the case of the polystyrene beads, and 140,330 and $2000 \mu \mathrm{m}$ in the case of the glass beads. The beads are washed in an ultrasound bath during 30 minutes and then dried. This is particularly important for experiments performed in Carbopol gels: when the unwashed beads are embedded into a Carbopol gel, it actually results in a lower yield stress than when the 
washed beads are suspended, indicating residual surface effects [2]; such residual surface effects may be due to colloidal impurities at the particle surface (or residual surfactant at the particle surface when polystyrene particles are used [2]). A single washing is enough to ensure a reproducible state. All materials were prepared (i) to ensure that the particle size is much larger than the paste microstructure size, (ii) to check that the results depend only on the mechanical properties of the paste i.e. that they are independent of the physicochemical origin of the yield stress, (iii) to check that the results are independent of the noncolloidal particles size (when the particles are monodisperse and have constant shape and surface texture), (iv) to check that there are neither particle/particle nor particle/paste physicochemical interactions. Moreover, by varying the suspending paste yield stress, it was checked that the dimensionless yield stress depends only on the particle volume fraction (when the particle are monodisperse). If we obtain the same behavior with suspensions prepared with all materials, including the cement pastes, and whatever the particle size, this ensures that there is no contribution from specific particles/material physicochemical interactions and that the results we obtain can be applied to the case of any other particles in any other yield stress fluid (in particular to any cement paste formulation).

The insertion of air is unavoidable. The effect of air on the yield stress is not negligible [2], it should thus be checked that its content is negligible: it changes not only the continuous phase mechanical properties [5] but also the effective bead volume fraction, which is a sensitive parameter at high volume fractions. However, methods such as centrifugation to remove the bubbles cannot be used if we want to ensure that the materials remain homogeneous as explained in Sec. 3. We thus chose to work with a constant volume of material in order to 
All the measurements we present in this paper were performed on suspensions of coarse particles embedded in pastes at a volume fraction $\phi$ ranging between 0 and $55 \%$, with an air content lower than $1 \%$.

\subsection{Rheological tools}

Most rheometric experiments are performed within a vane in cup geometry (inner radius $R_{i}=22.5 \mathrm{~mm}$, outer cylinder radius $R_{e}=45 \mathrm{~mm}$, height $H=$ $45 \mathrm{~mm}$ ) on a commercial rheometer (Bohlin C-VOR 200) that imposes either the torque or the rotational velocity (with a torque feedback). In order to avoid wall slip $[43,12]$, we use a six-blade vane as an inner tool, and we glue sandpaper on the outer cylinder wall. For the small particles in model yield stress fluids, we use another six-blade vane in cup geometry (inner radius $R_{i}=12.5 \mathrm{~mm}$, outer cylinder radius $R_{e}=18 \mathrm{~mm}$, height $\left.H=45 \mathrm{~mm}\right)$. Working within these wide-gap geometries allows for studying easily coarse particles and to ensure that, for all the materials studied, there are enough particles in the gap to consider that we measure the properties of a continuum medium (the suspension).

We measure the yield stress $\tau_{c}(\phi)$ of the paste as a function of the volume fraction $\phi$ of coarse particles embedded in the pastes. In a wide gap geometry, the shear stress $\tau$ continuously decreases within the gap: the shear stress at a radius $R$ is $\tau(R)=\frac{T}{2 \pi H R^{2}}$. Therefore, one has to choose a definition of the shear stress that is measured in a given rheological experiment. Here, we want to perform yield stress measurements; whatever the measurement 
method we choose, yield first occurs where the stress is maximal i.e. along the inner virtual cylinder. As consequence, we define the shear stress measurement as $\tau\left(R_{i}\right)=\frac{T}{2 \pi H R_{i}^{2}}$, so that the yield stress $\tau_{c}$ is correctly measured (any other definition of the shear stress would provide an underestimation of the yield stress). Anyway, we will focus on the evolution of the dimensionless yield stress $\tau_{c}(\phi) / \tau_{c}(0)$ with the bead volume fraction $\phi$, which should be independent of the definition of $\tau$.

\section{Experimental procedure}

In this section, we present the procedure aiming at showing the influence of the inclusion of coarse particles on the yield stress of cement pastes. We first show that the choice of the sample preparation and of the yield stress measurement procedure is critical to know how the particles are distributed in the suspension. We then establish a new procedure to ensure a good knowledge of the interstitial paste properties in the suspension.

\subsection{Preparation and yield stress measurement}

First, we need to define precisely the state of the materials we want study. Three points are actually important: (i) we want to perform our yield stress measurement on a homogeneous suspension, otherwise the measurement would have no meaning, (ii) we want to control the microstructure of the suspensions (i.e. the distribution of the neighbors of the coarse particles) to ensure that all measurements deal with the same state of the suspension, and can be compared and modelled, (iii) we need the interstitial cement paste to be 
These three points impose severe restrictions about the preparation and yield stress measurement procedures, as shown by Mahaut et al. [2]. They showed that measurements involving an important flow of the material (a large strain) pose several problems. First, a flow causes particle migration towards the low shear zones (the outer cylinder in coaxial cylinder geometries) i.e. creation of a heterogeneous structure. This migration phenomenon is well documented for suspensions of noncolloidal particles in Newtonian fluids [34-36] but is still badly known in yield stress fluids. As it needs a large strain to occur for moderate volume fraction [34,35], it may be avoided in these cases by performing only short duration experiments. However, for volume fractions of the order of $50 \%$ and more, migration is a critical phenomenon: it seems unavoidable as it is almost instantaneous as shown by Ovarlez et al. [36]. Another problem when suspensions flow is that an anisotropic microstructure of the particles is created by the flow, as observed in suspensions of particles in Newtonian fluids [38-40]. It is also a critical phenomenon: Mahaut et al. [2] showed that suspensions of isotropic and anisotropic microstructure have very different rheological properties.

These problems imply that we cannot preshear our materials with the rheometer and that we cannot use a yield stress measurement method based on a shear flow such as shear rate [8] or shear stress ramps [44] and creep tests [45]; we then have to measure the static yield stress. On the other hand, as the static yield stress of thixotropic materials depends on the time passed at rest in the solid state [46], the measurements have to be performed on a well defined state of the paste, i.e. the material needs to be first strongly presheared to get a destructured initial state. However, as pointed out above, we cannot apply 
a controlled preshear with the rheometer to the system after its preparation.

That is why, before loading the material in the measurement cup, we first presheared the cement paste alone during 2 minutes with the mixer at 285rpm; this ensures that the cement paste is initially in a destructured state. Then, the particles and the paste are mixed together in the measurement cylinder, and the loaded suspension is strongly stirred by hand in random directions to disperse the particles; this random stirring should ensure keeping the material in a destructured state while avoiding particle migration and anisotropy. Afterwards, the vane tool is inserted in the cup, and we perform our yield stress measurement after a given resting time with the vane method $[47,48]$ : a small rotational velocity, corresponding to a shear rate of $0.01 \mathrm{~s}^{-1}$ is imposed to the vane tool. Note that we checked that we observe the same effect of the particles on the yield stress whatever the low velocity that is chosen to drive the vane tool. Fig. 2 shows the shear stress vs. strain for yield stress measurement experiments performed in a cement paste. There is an overshoot, followed by a slow decrease of the shear stress: the peak defines the static yield stress, the decrease corresponds to destructuration of the material under the shear flow; the suspension structure at yield should then be isotropic and homogeneous. Then, any new yield stress measurement requires a new sample preparation or a new random manual preshear in the cup: it has been shown by Mahaut et al. [2] that the small strain of order 1 induced by the whole measurement procedure is sufficient to change the material state (it is sufficient to change the suspension microstructure or to induce migration): the suspension states before and after the yield stress measurements are characterized by different rheological properties. 
As we are interested in the influence of the particles on the yield stress of cement pastes, we will need to compare the suspension yield stress and the cement paste yield stress. It is thus important to ensure that we have a good knowledge of the properties of the interstitial paste state in the suspension. The procedure developed to ensure this measurement is presented in detail in the Appendix A. We present here the main steps.

First, we have to note that it is very difficult to achieve a good reproducibility of a cement paste mechanical behavior (see Appendix A). That is why we chose to work on the same batch for the measurement of the properties of the paste alone and for the suspension.

Then, for a given cement paste batch, we observe that yield stress measurements performed in the same conditions as regards thixotropic effects (i.e. for a 2 minutes resting time after a strong stirring of the paste) provide values that depend on the time $t_{\text {age }}$ elapsed since the constituents of the cement paste were mixed together (Appendix A). This means that one cannot know what is the yield stress of the interstitial paste in a suspension if the yield stress of the cement paste alone is not measured at exactly the same time after mixing as the yield stress of the suspension. That is why we chose to measure simultaneously the yield stress of the suspension and the yield stress of the cement paste alone in exactly the same conditions (same age $t_{\text {age }}$ after mixing the constituents of the cement paste, same time $t_{\text {rest }}$ after the end of the strong stirring), with the help of 2 rheometers that perform their measurements in parallel. 
We have also shown that when the same suspension sample, after a first resting period and a first measurement, is stirred again in the measurement cup, its interstitial cement paste is not in the same state of destructuration as the cement paste alone stirred with the same procedure (Appendix A). This means that the suspension and the cement paste cannot be compared anymore. A solution to this problem is to perform only a single measurement on a suspension, for a given resting time after its preparation.

A key point of the comparison between the suspension and the cement paste is actually that the cement paste is initially strongly presheared in the mixer for both samples: this defines an initial destructured state of the paste that is the same both for the interstitial cement paste and for the cement paste alone. After this preshear, the cement paste is loaded alone in one measurement cup, and with the particles in another cup. Both samples are then strongly stirred by hand during 30s in random directions: this ensures an homogeneous dispersion of the particles in the suspension, while keeping the cement paste in a destructured state in both samples. Then, the stirring is stopped simultaneously for both samples: this defines the beginning of the resting period. With this procedure, we have shown that the paste alone and the interstitial paste have the same history and thus the same behavior (see Appendix A).

Finally, as the cement paste is thixotropic, its static yield stress increases as a function of the time $t_{\text {rest }}$ elapsed since the end of the stirring. However, we showed that the yield stress value also depends on the time $t_{\text {age }}$ elapsed since the constituents were mixed together, even at short times. This would mean that a characterization of thixotropy would only have a meaning for this age $t_{\text {age }}$, and it would make the study of the impact of the coarse particles on this thixotropy difficult. Nevertheless, we have shown that the irreversible 
phenomena can be separated from the reversible phenomena. The thixotropic (reversible) increase of the yield stress is actually the same whatever the paste age $t_{\text {age }}$ : it depends only on the time $t_{\text {rest }}$ elapsed since the end of a preshear (Appendix A). The increase of the yield stress of our cement paste due to thixotropy is basically linear in $t_{\text {rest }}$ : it reads $\tau_{c}\left(t_{\text {rest }}\right)=A_{\text {thix }} t_{\text {rest }}$ with an increase rate $A_{\text {thix }}=12 \mathrm{~Pa} / \mathrm{min}$.

\subsection{Summary}

As a summary we present in Fig. 3 a sketch of the whole procedure used to study the influence of coarse particles on the yield stress of cement pastes.

This procedure ensures (i) that an homogeneous material is studied; (ii) that we study a well defined state of the material: we chose to study the case of isotropic distributions of particles; (iii) that the interstitial cement paste is well characterized; (iv) that the initial destructured state of the interstitial cement paste is well defined; (v) that thixotropy is accounted for and separated from irreversible phenomena; (vi) that the results obtained with cement pastes can be compared to measurements performed in other yield stress fluid.

\section{Experimental results}

In this section, we summarize the results of the yield stress measurements performed on the suspensions with the procedure presented above. We compare the results obtained with the cement pastes to the results obtained by Mahaut et al. [2] with various yield stress fluids, and to the Chateau-Ovarlez-Trung model [1]. 
In Fig. 4 we plot the dimensionless yield stress $\tau_{c}(\phi) / \tau_{c}(0)$ vs. the volume fraction $\phi$ of coarse particles embedded in the cement paste, when the yield stresses are measured with the procedure developed in Sec. 3 for various times $t_{\text {rest }}$ after the end of a strong stirring.

We first observe that the yield stress increases when the coarse particle volume fraction is increased. This increase is quite limited for volume fraction lower than 45\%: in this case, the yield stress is increased by a factor less than 3 . However, the yield stress is found to increase sharply at the approach of a $60 \%$ volume fraction. E.g., the yield stress of a suspension of $55 \%$ particles is 20 times higher than the yield stress of the interstitial cement paste.

We also observe in Fig. 4 that the same evolution of the yield stress with the particle volume fraction is found whatever the time $t_{\text {rest }}$ passed at rest before the measurement. This means that the yield stress of suspensions of coarse particles embedded at a volume fraction $\phi$ in a thixotropic cement paste of time-dependent yield stress $\tau_{c}(0, t)$ reads

$$
\tau_{c}(\phi, t)=\tau_{c}(0, t) g(\phi)
$$

This feature is expected if the coarse (i.e. noncolloidal) particles have only a mechanical interaction with the cement paste [2]: in this case, they should not interfere with the physical process at the origin of thixotropy. Then, at time $t$ the interstitial paste has naturally the same yield stress $\tau_{c}(0, t)$ as if it had not been in contact with the coarse particles. Finally, as the relative increase 
of the yield stress due to the monodisperse particles should be a function of their volume fraction $\phi$ only, independently of the value of the interstitial fluid yield stress, the yield stress of the suspension at time $t$ is expected to be equal to $\tau_{c}(0, t)$ multiplied by some function $g(\phi)$ whatever $\tau_{c}(0, t)$, as observed experimentally.

Eq. 1 has an interesting consequence: it means that it is sufficient to know how the interstitial cement paste evolves in time to predict the suspension evolution at rest. This is important for fresh concrete as their behavior is hard to measure: our results show that the knowledge of the cement paste structuration rate at rest is sufficient to predict the fresh concrete structuration rate. As found on the cement paste we studied (see Sec. 3.2) the yield stress evolution at rest after a preshear of a cement paste usually reads [19]:

$$
\tau_{c}(0, t)=\tau_{c}(0)+A_{t h i x} t
$$

where $A_{\text {thix }}$ is the structuration rate of the paste. In this case, Eq. 1 reads:

$$
\tau_{c}(\phi, t)=\tau_{c}(0) g(\phi)+A_{t h i x} g(\phi) t
$$

As a consequence, if the mechanical impact of the coarse particles is to increase the yield stress by a factor $g(\phi)$, then their impact on the structuration rate of the paste is to increase it also by a factor $g(\phi)$. It is thus sufficient to measure the cement paste yield stress evolution in time (i.e. $A_{\text {thix }}$ ) and to measure the increase of the yield stress with the volume fraction (i.e. $g(\phi))$ for a single resting time $t_{\text {rest }}$ to infer the value $A_{\text {thix }} g(\phi)$ of the structuration rate of the suspension (and more generally of fresh concrete). 
In Fig. 5, we plot a summary of the dimensionless yield stress measurements $\tau_{c}(\phi) / \tau_{c}(0)$ performed on all the materials by Mahaut et al. [2], together with the results obtained with cement pastes.

We find that all the results are consistent: the dimensionless yield stress $\tau_{c}(\phi) / \tau_{c}(0)$ is independent of the physicochemical origin of the material yield stress, of the bead material and of the bead size, and of the paste yield stress; it is a function of the volume fraction only. This means that the particles have a purely mechanical contribution to the paste behavior, which is independent of the physicochemical properties of the materials: the only important matter is the value of the yield stress of the paste. This also validates our approach: as long as the coarse particle size is much larger than the cement paste microstructure, a suspension of coarse particles in a cement paste can be considered more generally as a suspension of rigid noncolloidal particles in a yield stress fluid.

This result helps proposing a method than can be applied to obtain quickly the effect of particles of any kind (any shape, any size distribution) on the yield stress of a cement paste. Actually, preparing a model yield stress fluid of stable and reproducible rheological properties, showing no setting nor thixotropic effects (e.g. an emulsion), is quite easy, and measurements are much easier to perform on these materials. Then a great amount of accurate experiments can be performed to measure the properties of suspensions of particles in this yield stress fluid. Finally, the result of the measurement of the dimensionless yield stress $\tau_{c}(\phi) / \tau_{c}(0)=g(\phi)$ as a function of the volume fraction $\phi$ of particles 
in this yield stress fluid should hold if the interstitial paste is a cement paste.

Moreover, we have shown that the knowledge of the structuration rate $A_{\text {thix }}$ of a cement paste is sufficient to infer the structuration rate of the suspension of particles in this cement paste (it is equal to $A_{\text {thix }} g(\phi)$ ). A measurement of the cement paste structuration at rest plus the measurement $\tau_{c}(\phi) / \tau_{c}(0)=g(\phi)$ in a model yield stress fluid then provides everything that is needed to infer the behavior of mortars or concretes. Note however that these results apply only as long as the particle size is much larger than the cement paste microstructure typical size so that the particles see the yield stress fluid as an homogeneous material. This should not be true otherwise: if the particles were to be sensitive to the cement paste microstructure, then the behavior should depend on the exact details of the specific microstructure of each paste. E.g., in the case of particles suspended in a foam, Cohen-Addad et al. [49] found that the behavior of the suspension depends on the particle size for particles of size lower than 5 times the bubble size in the foam. Note finally that another important requirement is that the fraction of superplasticizer adsorbed at the surface of the aggregates suspended in the paste is negligible. The study of Hammer and Wallevik [23] suggests that in some cases (it may depend strongly on the cement paste composition) this may be true only if the aggregates are larger than 0.25 to $0.5 \mathrm{~mm}$; in such cases, our approach would then be valid for SCC only if the suspending yield stress fluid includes the fine aggregates (of size lower than 0.25 to $0.5 \mathrm{~mm}$ in the study of Hammer and Wallevik). 
Proposing a theoretical value for the dimensionless yield stress is challenging. However, it has been shown by Chateau et al. [1] that it is possible to give a general relationship between the linear response of the materials (e.g. its dimensionless elastic modulus $G^{\prime}(\phi) / G^{\prime}(0)$ as probed under the yield stress) and the dimensionless yield stress $\tau_{c}(\phi) / \tau_{c}(0)$ of a suspension of rigid particles in a yield stress fluid. This estimate is based on the following hypotheses: the particles are rigid and noncolloidal; there are no physicochemical interactions between the particles and the paste; the distribution of the particles is isotropic. This is what we have managed to perform experimentally, therefore, our experiments are fitted to provide a test of these theoretical predictions. Chateau et al. [1] find

$$
\tau_{c}(\phi) / \tau_{c}(0)=\sqrt{(1-\phi) G^{\prime}(\phi) / G^{\prime}(0)}
$$

Mahaut et al. [2] have measured the elastic modulus of all the suspensions studied above, and found a Krieger-Dougherty model to apply $G^{\prime}(\phi) / G^{\prime}(0)=$ $\left(1-\phi / \phi_{m}\right)^{-2.5 \phi_{m}}$ for the dimensionless elastic modulus. Combining this equation and the theoretical expression Eq. 4 thus yields for the yield stress the Chateau-Ovarlez-Trung model [1]

$$
\frac{\tau_{c}(\phi)}{\tau_{c}(0)}=\sqrt{\frac{1-\phi}{\left(1-\phi / \phi_{m}\right)^{2.5 \phi_{m}}}}
$$

which should be valid for any isotropic suspension of rigid spherical noncolloidal particles in yield stress fluids with no physicochemical interactions between the particles and the paste. 
Our experimental data are compared to Eq. 5 on Fig. 5. We find a remarkable agreement between our data and this model with a best fit for $\phi_{m}=0.56$; note that this value of 0.56 is valid only for the case of monodisperse spherical particles we studied.

Note however that Eq. 5 can a priori be easily modified to account for polydispersity and for complex shapes of the particles when studying more complex suspensions. Actually, Eq. 4 should hold in all cases. It is then sufficient to know what is the linear response of a suspension made with the studied particles to infer the yield stress value. This linear response can be measured with the method presented in this paper (it is the dimensionless elastic modulus $\left.G^{\prime}(\phi) / G^{\prime}(0)\right)$; it can also be inferred from the huge amount of dimensionless viscosity data from the literature dealing with suspensions of particles in Newtonian fluids (with the same particles): the problem of the elasticity of a suspension of rigid particles in a linear elastic material is actually formally similar to the problem of the viscosity a suspension of rigid particles in a Newtonian (thus linear) material.

\subsection{Comments}

Our results are naturally close to the Ancey and Jorrot [33] ones, as they have chosen to measure the yield stress of the suspension by means of a slump test which ensures avoiding migration of particles and anisotropy of the material. On the other hand, we find very different values from Geiker et al. [26], Erdogan [27], and Toutou and Roussel [28]. As pointed out in Sec. 1 and Sec. 3, this is due to the shortcomings of their experimental procedure which is based on a flow and an extrapolation of the dynamic yield stress from a flow curve. Their 
materials are then likely to be heterogeneous and anisotropic. Moreover, an extrapolation from a flow curve provides an overestimation of the yield stress of the suspension [1] because the suspension departs from a Herschel-Bulkley (or Bingham) model at very low shear rate (unaccessible to most concrete rheometers) and has a lower yield stress than the one extrapolated from the accessible flow curve, even if the suspending yield stress fluid has a HerschelBulkley (or Bingham) behavior.

Finally, note that in most papers the results are presented vs. $\phi / \phi_{\max }$ where $\phi_{\max }$ is the maximum packing fraction (taken at about 0.65 for monodisperse particles) and the yield stress divergence is expected to occur for $\phi / \phi_{\max }=1$.

This is not correct: the maximum volume fraction $\phi_{m}$ for the yield stress sharp increase should not be taken as the maximum volume fraction one can reach by packing particles together (which is the definition of the maximum packing fraction $\left.\phi_{\max }\right)$. The maximum volume fraction for the yield stress sharp increase is rather the one at which direct contacts become important, which is the limit of application of models including only hydrodynamic interactions between the particles, and also the limit between SCC and ordinary rheology concretes [29]. This explains why we find the yield stress to diverge at around $56 \%$ while the maximum packing fraction is of about $65 \%$ for spheres.

\section{Conclusion}

We have studied the behavior of suspensions of coarse particles in a thixotropic cement paste. We managed to design procedures that allow for (i) studying an homogeneous and isotropic suspension, (ii) comparing the yield stress of a given cement paste to that of the same cement paste added with particles, 
(iii) accounting properly for the thixotropy of the cement paste. We observed that the yield stress of these pastes follows the very simple Chateau-OvarlezTrung model $[1] \tau_{c}(\phi) / \tau_{c}(0)=\sqrt{(1-\phi)\left(1-\phi / \phi_{m}\right)^{-2.5 \phi_{m}}}$, with $\phi_{m}=0.56$ for monodisperse spherical particles, consistently with the experimental results of Mahaut et al. [2] obtained with many different suspensions. This supports the fact that the yield stress of the suspension is independent of the physicochemical properties of the yield stress fluid, and depends only on its yield stress value. This shows that studies of suspensions in model yield stress fluids can be used as a general tool to infer the behavior of fresh concrete. Moreover, we showed that the thixotropic structuration rate of these pastes (their static yield stress increase rate in time) is not changed by the presence of the particles. This shows that it is sufficient to measure the cement paste yield stress evolution in time and to measure the increase of the yield stress with the volume fraction of coarse particles for a single resting time to predict the value of the structuration rate of fresh concrete. For a linear increase of the cement paste yield stress with a rate $A_{\text {thix }}$, we predict a linear increase of the suspension with a rate $A_{t h i x} \sqrt{(1-\phi)\left(1-\phi / \phi_{m}\right)^{-2.5 \phi_{m}}}$.

\section{A Characterization of the interstitial paste}

In this appendix, we detail the arguments that have led to develop the procedure presented in Sec. 3. This new procedure is built to ensure a good knowledge of the mechanical properties and of the state of structuration of the interstitial cement paste in the suspension.

First, we have to note that it is very difficult to achieve a good reproducibility of a fresh cement paste mechanical behavior. In Fig. A.1a we show the result 
of the yield stress measurements performed (apparently) exactly in the same conditions in 2 cement pastes having the same composition. We observe that the uncertainty on the yield stress of the cement paste we get is of order $25 \%$. This means that, if we want to measure accurately the ratio of the suspension yield stress to the interstitial cement paste yield stress, we cannot compare the properties of suspensions of particles in a cement paste to the properties of a cement paste having the same composition but being from a different batch. That is why we chose to work on the same batch for the measurement of the properties of the paste alone and for the suspension.

Then, for a given cement paste batch, we could propose to first measure the cement paste yield stress and then the suspension yield stress. For the results to be comparable, one would then just have to perform the experiment in the same conditions as regards thixotropy (i.e. for the same resting time after a strong preshear). In order to check the validity of this method, we performed yield stress measurements several times in the same conditions (i.e. for a 2 minutes resting time after a strong stirring of the paste) on a single cement paste batch. The results of this experiment are depicted in Fig. A.2 as a function of the time $t_{\text {age }}$ elapsed since the constituents of the cement paste were mixed together. We observe that due to various irreversible chemical interactions in the material, the cement paste yield stress, measured in the same conditions as regards thixotropic effects, evolves (non-monotonously) as a function of the time $t_{\text {age }}$ elapsed since the constituents of the cement paste were mixed together. This means that one cannot know what is the yield stress of the interstitial paste in a suspension if the yield stress of the cement paste alone is not measured at exactly the same time after mixing as the yield stress of the suspension. That is why we chose to measure simultaneously the 
yield stress of the suspension and the yield stress of the cement paste alone in exactly the same conditions (same age $t_{\text {age }}$ after mixing the constituents of the cement paste, same time $t_{r e s t}$ after the end of the strong stirring), with the help of 2 rheometers that perform their measurements in parallel. We show in Fig. A.1b that, as expected, this method yields a very low uncertainty when the measurements are performed on the same cement paste (without particles).

Now, by performing these simultaneous measurements of the suspension yield stress $\tau_{c}(\phi)$ and of the cement paste yield stress $\tau_{c}(0)$ several times, at various ages $t_{\text {age }}$ after mixing the constituents of the cement paste, we should observe the same effect of the particles on the yield stress whatever the age of the cement paste. In Fig. A.3 we present the dimensionless yield stress $\tau_{c}(\phi) / \tau_{c}(0)$ as a function of the time $t_{\text {age }}$ elapsed since the constituents of the cement paste were mixed together. We observe that $\tau_{c}(\phi) / \tau_{c}(0)$ is not constant in time. This means that the interstitial paste is not in the same mechanical state as the paste alone although they have apparently the same history. The only difference stands in the preshear procedure: before performing each measurement, the paste and the suspension are presheared to ensure a reproducible destructured initial state. As pointed out above, the preshear has to be manual to avoid migration and anisotropy. Our results show that this preshear is not as efficient in the suspension as in the cement paste. It is harder to shear the suspension, thus an experimentalist cannot shear the suspension the same way as the paste alone. It can be noted that, in the case of a strong mechanical preshear in a mixer, an opposite result has been obtained by Toutou and Roussel [28] due to the mixing effect of the particles. As a result of these imperfect and perturbing preshears, the differences between the structuration state of 
the paste alone and of the interstitial paste in the suspension increases with time. The suspension and the cement paste cannot be compared anymore, and the function $\tau_{c}(\phi) / \tau_{c}(0)$ is no more correctly measured by this means. A solution to this problem is to perform only a single measurement on a suspension, for a given resting time after its preparation. As pointed out in Sec. 3.2, a key point of the comparison between the suspension and the cement paste is then that the cement paste alone is first initially strongly presheared in the mixer for both samples before being loaded (and eventually mixed with the particles) in the measurement cups: this defines an initial destructured state of the paste that is the same both for the interstitial cement paste and for the cement paste alone. The manual stirring in the measurement cup then ensures an homogeneous dispersion of the particles in the suspension, while keeping the cement paste in a destructured state in both samples. With this procedure, one ensures that the paste alone and the interstitial paste have the same history and thus the same behavior. We show actually in Fig. A.3 that, in these conditions, the same value of $\tau_{c}(\phi) / \tau_{c}(0)$ is found within the measurement uncertainty whatever the time $t_{\text {age }}$ elapsed since the constituents of the cement paste were mixed together.

Finally, as the cement paste is thixotropic, its static yield stress increases as a function of the time $t_{\text {rest }}$ elapsed since the end of the stirring. However, if we want to account properly for the (reversible) thixotropic behavior of the cement paste, and to check what the influence of the particles on this thixotropic behavior is, we face a problem. We showed that the value of the yield stress measured 2 minutes after a strong stirring evolves as a function of the time $t_{\text {age }}$ elapsed since the constituents were mixed together, even at short times. This would mean that in order to characterize the increase of 
the yield stress of cement pastes due to structuration at rest, as a function of the resting time $t_{\text {rest }}$ after a strong stirring, we would need to perform all the yield stress measurements only at a same given age $t_{\text {age }}$ after mixing the constituents of the cement paste. And this characterization of thixotropy would only have a meaning for this age $t_{\text {age }}$. However, we show in the following that the thixotropic increase of the yield stress is actually the same whatever the paste age. In Fig. A.4a, we plot the yield stress of a cement paste as a function of the age $t_{\text {age }}$ of the paste for 3 different times $t_{\text {rest }}$ after a strong stirring; note that as we have only 2 rheometers, these measurements had to be performed on 2 batches, so that the uncertainties may be rather large (as in Fig. A.1a).

We observe the same evolution of the paste behavior as a function of $t_{\text {age }}$ whatever $t_{\text {rest }}$. An important consequence is that the irreversible effects can be separated from the reversible effects by writing

$$
\tau_{c}\left(t_{\text {rest }}, t_{\text {age }}\right)=\tau_{c}\left(t_{\text {age }}\right)+\tau_{c}\left(t_{\text {rest }}\right)
$$

where $\tau_{c}\left(t_{\text {age }}\right)$ is the yield stress that would be measured just after a preshear, which depends on the time $t_{\text {age }}$ elapsed since the constituents were mixed together, and $\tau_{c}\left(t_{\text {rest }}\right)$ represents the increase of the yield stress due to thixotropic effects, which depends only on the time elapsed since the end of a preshear. This is shown in Fig. A.4b: all data are superposed when shifted by a constant value that depends only on $t_{\text {rest }}$. From the superposition of data in Fig. A.4b, we find that $\tau_{c}\left(t_{\text {rest }}=4 \mathrm{~min}\right)-\tau_{c}\left(t_{r e s t}=2 \mathrm{~min}\right)=24 \mathrm{~Pa}$ and $\tau_{c}\left(t_{\text {rest }}=6 \mathrm{~min}\right)-\tau_{c}\left(t_{\text {rest }}=2 \mathrm{~min}\right)=48 \mathrm{~Pa}$. This is consistent with the simple law proposed by Roussel [19] i.e. the increase of the yield stress due to thixotropy 
${ }_{745}$ is basically linear in $t_{\text {rest }}$ : it reads $\tau_{c}\left(t_{\text {rest }}\right)=A_{\text {thix }} t_{\text {rest }}$ with an increase rate $746 A_{\text {thix }}=12 \mathrm{~Pa} / \mathrm{min}$. Finally, as the absolute increase of the yield stress due to 747 thixotropic effects is the same at any time $t_{\text {age }}$ (lower than $90 \mathrm{~min}$ ) since the 748 constituents were mixed together, this shows that studies of thixotropy and 749 of the effect of the coarse particles on this thixotropy performed at different 750 751 times $t_{\text {age }}$ can be compared together and provide relevant information on the thixotropy of the suspensions. 
[1] X. Chateau, G. Ovarlez, K.L. Trung, Homogenization approach to the behavior of suspensions of noncolloidal particles in yield stress fluids, J. Rheol. (2008) 52 $489-506$.

[2] F.Mahaut, X.Chateau, P.Coussot, G.Ovarlez, Yield stress and elastic modulus of suspensions of noncolloidal particles in yield stress fluids, J. Rheol. (2008) 52 $287-313$.

[3] G.H. Tatersall, P.G.F. Banfill, The Rheology of Fresh Concrete, Pitman, London, 1983.

[4] L. Nachbaur, J.C. Mutin, A. Nonat, L. Choplin, Dynamic mode rheology of cement and tricalcium silicate pastes from mixing to setting, Cem. Concr. Res. 31 (2001) 183-192.

[5] R.G. Larson, The structure and rheology of complex fluids, Oxford University Press, New York, 1999.

[6] Y. Otsubo, S. Miyai, K. Umeya, Time-dependant flow of cement pastes, Cem. Concr. Res. 10 (1980) 631-638.

[7] P.F.G. Banfill, D.C. Saunders, On the viscosimetric examination of cement pastes, Cem. Concr. Res. 11 (1981) 363-370.

[8] M.R. Geiker, M. Brandl, L.N. Thrane, D.H. Bager, O. Wallevik, The effect of measuring procedure on the apparent rheological properties of self compacting concrete, Cem. Concr. Res. 32 (2002) 1791-1795 .

[9] J. Assaad, K.H. Khayat, H. Mesbah, Assessment of thixotropy of flowable and self-consolidating concrete, ACI Materials Journal 100 (2003) 99-107.

[10] N. Roussel, Steady and transient flow behaviour of fresh cement pastes, Cem. Concr. Res. 35 (2005) 1656-1664. 
[11] P. Billberg, Development of SCC static yield stress at rest and its effect on the lateral form pressure, in: S.P. Shah (Ed.), Proceedings of the Second North American Conference on the Design and use of Self-Consolidating Concrete and the Fourth International RILEM Symposium on Self-Compacting Concrete, Chicago, 2005.

[12] P. Coussot, Rheometry of Pastes, Suspensions and Granular Materials, John Wiley \& Sons, New York, 2005.

[13] N. Roussel, Rheology of fresh concrete: from measurements to predictions of casting processes, Materials and Structures 40 (2007) 1001-1012.

[14] J. Assaad, K.H. Khayat, Variations of lateral and pore water pressure of selfconsolidating concrete at early age, ACI Materials Journal 101 (2004) 310-317.

[15] K.H. Khayat, J. Assaad, H. Mesbah, M. Lessard, Effect of section width and casting rate on variations of formwork pressure of self-consolidating concrete, Materials and Structures 38 (2005) 73-78.

[16] G. Ovarlez, N. Roussel, A physical model for the prediction of lateral stress exerted by self-compacting concrete on formwork, Materials and Structures 37 (2006) 269-279.

[17] J.C. Tchamba, S. Amziane, G. Ovarlez, N. Roussel, Lateral stress exerted by fresh fluid concrete on formwork: laboratory experiments, Cem. Concr. Res. 38 (2008) 459-466.

[18] N. Roussel, A theoretical frame to study stability of fresh concrete, Materials and Structures 39 (2006) 75-83.

[19] N. Roussel, Thixotropy model for fresh fluid concretes: theory, validation and applications, Cem. Concr. Res. 36 (2006) 1797-1806.

[20] C.F. Ferraris, L.E. Brower (Eds), Comparison of concrete rheometers: 
International tests at LCPC (Nantes, France) in October 2000, National Institute of Standards and Technology Internal Report 6819, 2001.

[21] R. Flatt, Towards a prediction of superplasticized concrete rheology, Materials and structures 27 (2004) 289-300 .

[22] J.J. Stickel, R.L. Powell, Fluid Mechanics and Rheology of Dense Suspensions, Annu. Rev. Fluid Mech. 37 (2005) 129-149 .

[23] T.A. Hammer, J.E. Wallevik, On the correlation between rheology of paste, mortar and concrete, in: S.P. Shah (Ed.), Proceedings of the Second North American Conference on the Design and use of Self-Consolidating Concrete and the Fourth International RILEM Symposium on Self-Compacting Concrete, Chicago, 2005.

[24] F.de Larrard, Concrete Mixture Proportioning, Spon Press, London, 1999.

[25] F. de Larrard, T. Sedran, Mixture proportioning of high performance concrete, Cem. Concr. Res. 32 (2002) 1699-1704.

[26] M.R. Geiker, M.Brandl, L.N. Thrane, L.F. Nielsen, On the effect of coarse aggregate fraction and shape on the rheological properties of self-compacting concrete, Cement Concrete and Aggregates 24 (2002) 3-6.

[27] T.S. Erdogan, Determination of aggregate shape properties using X-ray tomographic methods and the effect of shape on concrete rheology, $\mathrm{PhD}$ thesis, University of Texas at Austin, 2005.

[28] Z. Toutou, N. Roussel, Multi scale experimental study of concrete rheology: from water scale to gravel scale, Materials and Structures 37 (2006) 167-176.

[29] Y.M. Joumana, M. Chaouche, M. Guerinet, M. Moranville, N. Roussel, From ordinary rhelogy concrete to self compacting concrete: a transition between frictional and hydrodynamic interactions, submitted to Cem. Concr. Res. (2008). 
[30] L.F. Nielsen, Rheology of some fluid extreme composites such as fresh selfcompacting concrete, Nordic Concrete Resarch 2 (2001) 83-93.

[31] D.A. Williams, A.W. Saak, H.M. Jennings, The influence of mixing on the rheology of fresh cement paste, Cem. Concr. Res. 29 (1999) 14911496.

[32] P. Coussot, Mudflow Rheology and Dynamics, Balkema, Rotterdam, 1997.

[33] C. Ancey, H. Jorrot, Yield stress for particle suspensions within a clay dispersion, J. Rheol. 45 (2001) 297-319.

[34] D. Leighton, A. Acrivos, The shear-induced migration of particles in concentrated suspensions, J. Fluid Mech. 181 (1987) 415-439.

[35] R.J. Phillips, R.C. Armstrong, R.A. Brown, A.L. Graham, J.R. Abbott, A constitutive equation for concentrated suspensions that accounts for shearinduced particle migration, Phys. Fluids 4 (1992) 30-40.

[36] G. Ovarlez, F. Bertrand, S. Rodts, Local determination of the constitutive law of a dense suspension of noncolloidal particles through magnetic resonance imaging, J. Rheol. 50 (2006) 259-292.

[37] N. Roussel, P. Coussot, Fifty-cent rheometer for yield stress measurements: From slump to spreading flow, J. Rheol. 49 (2005) 705-718.

[38] F. Gadala-Maria, A. Acrivos, Shear-induced structure in a concentrated suspension of solid spheres, J. Rheol. 24 (1980) 799-814.

[39] F. Parsi, F. Gadala-Maria, Fore-and-aft asymmetry in a concentrated suspension of solid spheres, J. Rheol. 31 (1987) 725-732.

[40] A. Sierou, J.F. Brady, Rheology and microstructure in concentrated noncolloidal suspensions, J. Rheol. 46 (2002) 1031-1056.

[41] R.J. Ketz, R.K. Prud'homme, W.W. Graessley, Rheology of concentrated microgel solutions, Rheol. Acta 27 (1988) 531-539. 
853 [42] J.O. Carnali, M.S. Naser, The use of dilute suspension viscosimetry to ${ }_{854}$ characterize the network properties of Carbopol microgels, Colloid Polym. Sci. 270 (1992) 183-193.

[43] A.W. Saak, H.M. Jennings, S.P. Shah, The influence of wall slip on yield stress and visco-elastic measurements of cement pastes, Cem. Concr. Res. 31 (2001) 205212.

[44] P.H.T. Uhlerr, J. Guo, C. Tiu, X.M. Zhang, J.Z.Q. Zhou, T.N. Fang, The shearinduced solid-liquid transition in yield stress materials with chemically different structures, J. Non-Newtonian Fluid Mech. 125 (2005) 101-119 .

[45] P. Coussot, H. Tabuteau, X. Chateau, L. Tocquer, G. Ovarlez, Aging and solid or liquid behavior in pastes, J. Rheol 50 (2006) 975-994.

[46] D.C.H. Cheng, Yield stress: A time-dependent property and how to measure it, Rheol. Acta 25 (1986) 542-554.

[47] Q.D. Nguyen, D.V. Boger, Direct yield stress measurement with the vane method, J. Rheol. 29 (1985) 335-347.

[48] P.V. Liddell, D.V. Boger, Yield stress measurements with the vane, J. NonNewtonian Fluid Mech. 63 (1996) 235-261.

[49] S. Cohen-Addad, M. Krzan, R. Höhler, B. Herzhaft, Rigidity percolation in particle laden foams, Phys. Rev. Lett. 99 (2007) 168001. 
Table 1

\begin{tabular}{|c|c|}
\hline Constituents & $\%$ by mass \\
\hline $\mathrm{SiO}_{2}$ & 20.95 \\
\hline $\mathrm{Al}_{2} \mathrm{O}_{3}$ & 4.08 \\
\hline $\mathrm{TiO}_{2}$ & 0.14 \\
\hline $\mathrm{Fe}_{2} \mathrm{O}_{3}$ & 0.22 \\
\hline $\mathrm{CaO}$ & 65.55 \\
\hline $\mathrm{MgO}$ & 0.49 \\
\hline $\mathrm{Na}_{2} \mathrm{O}$ & 0.12 \\
\hline $\mathrm{K}_{2} \mathrm{O}$ & 0.20 \\
\hline $\mathrm{SO}_{3}$ & 2.60 \\
\hline RI & 1.47 \\
\hline PAF & 3.36 \\
\hline
\end{tabular}

Cement chemical constituents. 


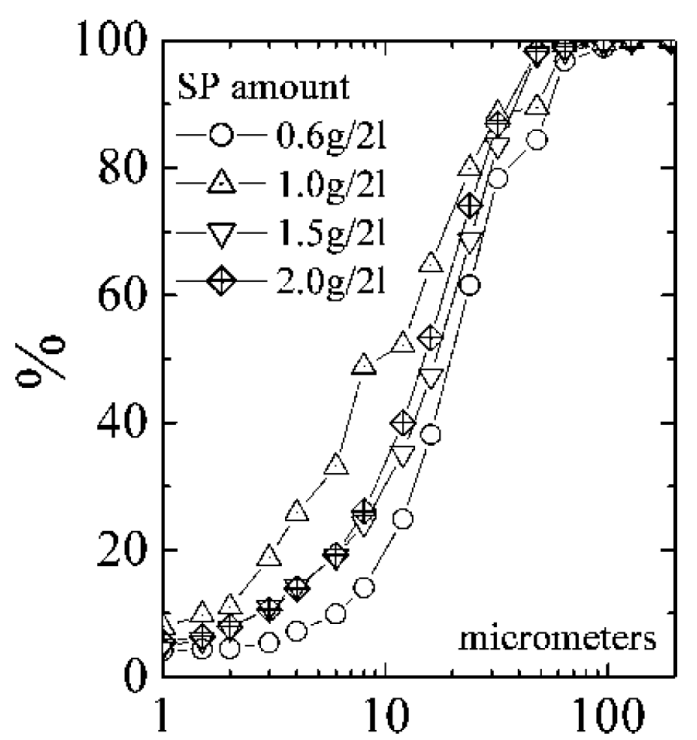

Fig. 1. Cement size distribution curve for various superplasticizer (SP) amount.

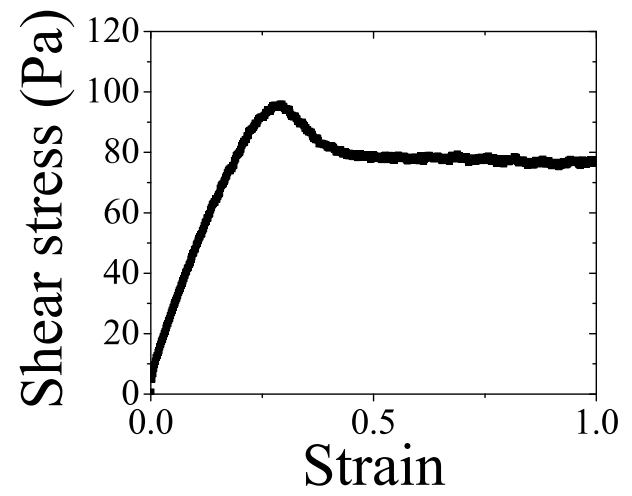

Fig. 2. Shear stress vs. strain when slowly shearing a cement paste at $10^{-2} \mathrm{~s}^{-1}$ 2 minutes after a strong stirring of the paste. 


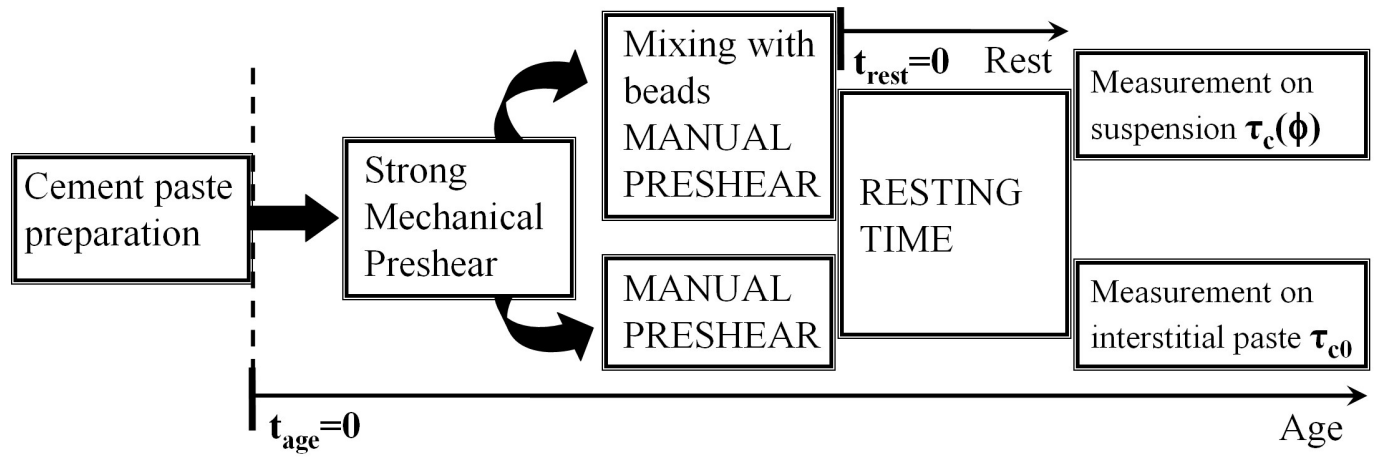

Fig. 3. Sketch of the procedure designed to study the evolution of the dimensionless yield stress $\tau_{c}(\phi) / \tau_{c}(0)$ with the volume fraction $\phi$ of particles in the suspension.

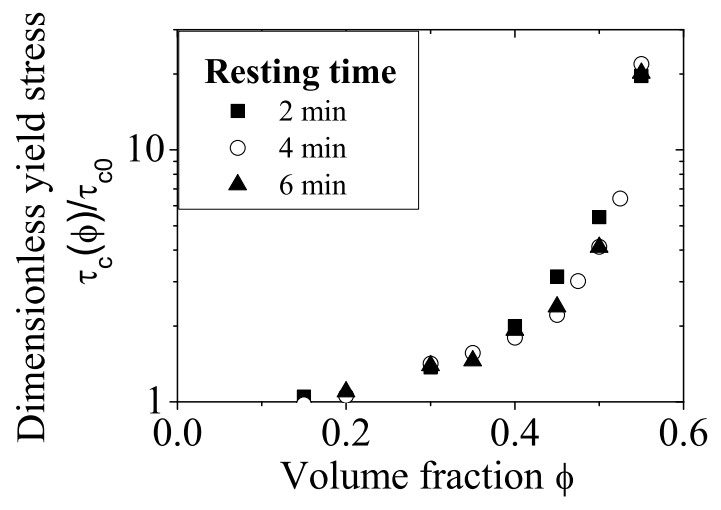

Fig. 4. Dimensionless yield stress $\tau_{c}(\phi) / \tau_{c}(0)$ vs. the bead volume fraction $\phi$ for suspensions of $2 \mathrm{~mm}$ glass beads in a cement paste, measured with the procedure developed in Sec. 3 for various times $t_{r e s t}$ after a strong stirring of the suspension. 


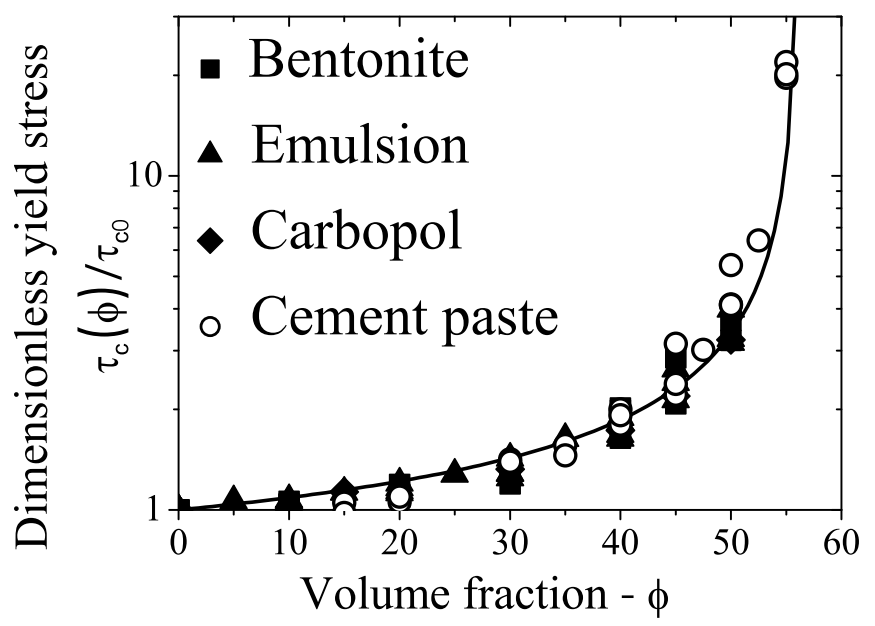

Fig. 5. Dimensionless yield stress $\tau_{c}(\phi) / \tau_{c}(0)$ vs. the bead volume fraction $\phi$ for suspensions of 80,140 , and $315 \mu \mathrm{m}$ polystyrene beads and $140 \mu \mathrm{m}, 330 \mu \mathrm{m}$ and $2 \mathrm{~mm}$ glass beads in various bentonite suspensions, emulsions and Carbopol gels (results from Mahaut et al. [2]), and for $2 \mathrm{~mm}$ glass beads suspended in a cement paste. The solid line is the Chateau-Ovarlez-Trung model $\sqrt{(1-\phi) \times\left(1-\phi / \phi_{m}\right)^{-2.5 \phi_{m}}}$ with $\phi_{m}=0.56$.
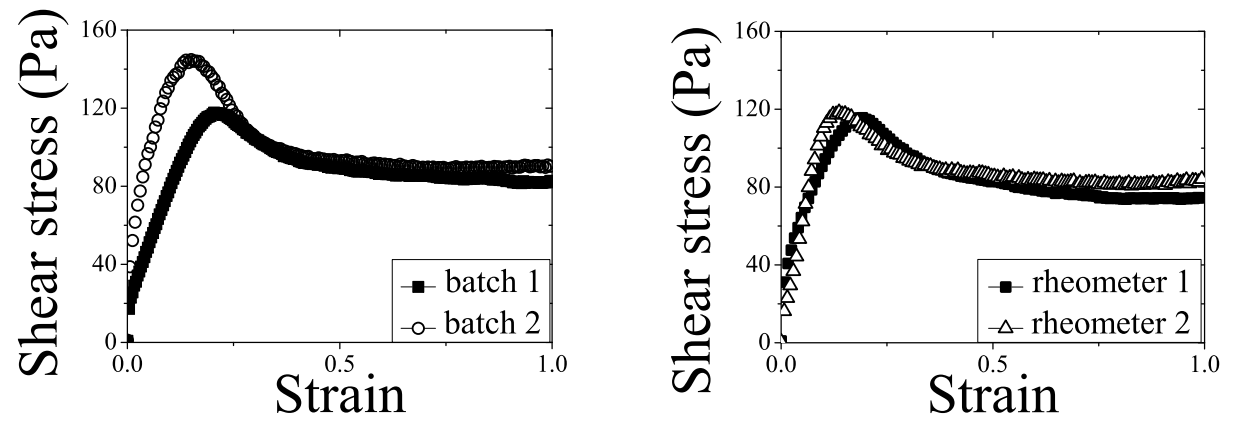

Fig. A.1. a) Shear stress vs. strain when slowly shearing two batches of a cement paste at $10^{-2} \mathrm{~s}^{-1} 2$ minutes after a strong stirring of the paste. b) Shear stress vs. strain when slowly shearing simultaneously on 2 rheometers a cement paste from a single batch at $10^{-2} \mathrm{~s}^{-1} 2$ minutes after a strong stirring of the paste. 


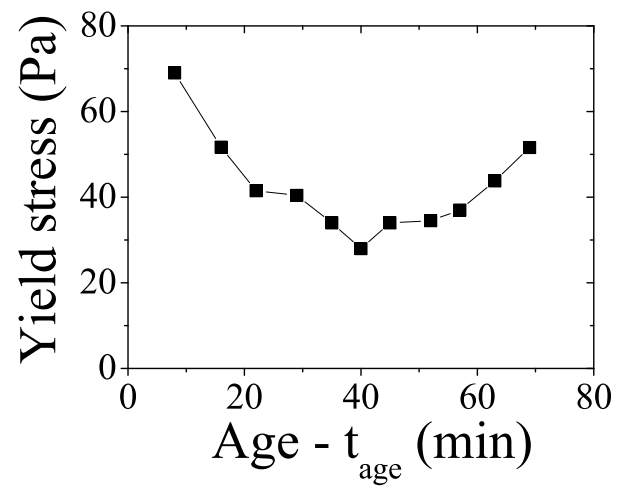

Fig. A.2. Yield stress of a cement paste measured 2 minutes after a strong stirring of the paste vs. the time $t_{\text {age }}$ elapsed since the constituents of the cement paste were mixed together.

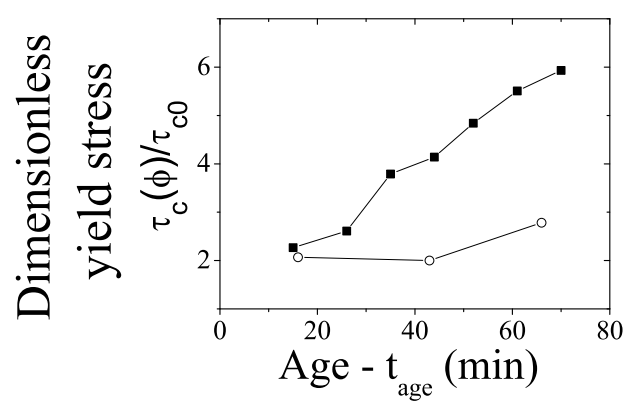

Fig. A.3. Dimensionless yield stress $\tau_{c}(\phi) / \tau_{c}(0)$ measured 2 minutes after a strong stirring of the suspension vs. the time $t_{\text {age }}$ elapsed since the constituents of the cement paste were mixed together (with a volume fraction of coarse particles $\phi=40 \%$ ), in two cases: when the same suspension of particles is used for all measurements (squares); when the particles are mixed with the cement paste just before each measurement (open circles). 

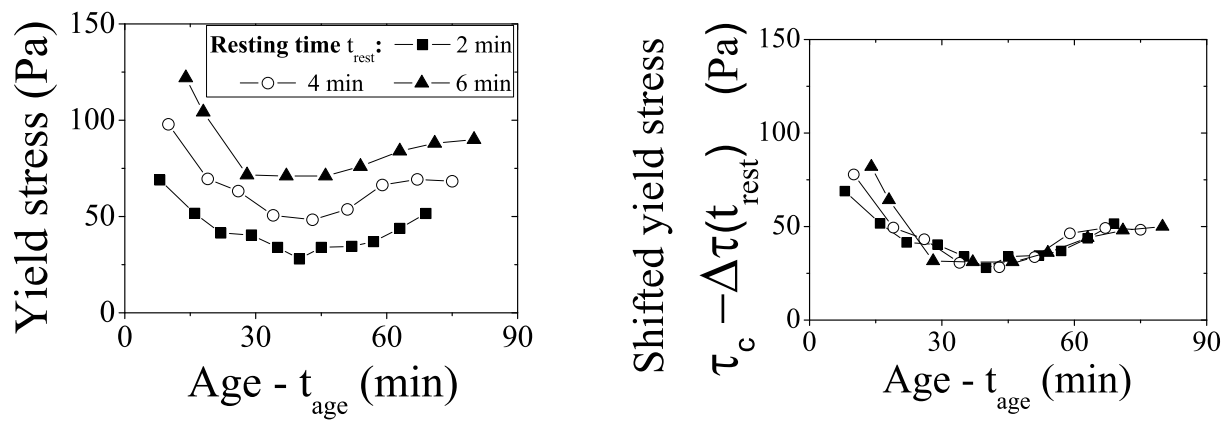

Fig. A.4. a) Yield stress of a cement paste measured 2, 4 and 6 minutes after a strong stirring of the paste vs. time $t_{\text {age }}$ elapsed since the constituents of the cement paste were mixed together. b) Data of Fig. A.4a rescaled by shifting the yield stress values by a function $\Delta \tau_{c}\left(t_{r e s t}\right)$ of the time $t_{r e s t}$ elapsed since the end of the strong stirring. 